\title{
Gaze aversion: A response to cognitive or social difficulty?
}

\author{
G. DOHERTY-SNEDDON and F. G. PHELPS \\ University of Stirling, Stirling, Scotland
}

\begin{abstract}
When asked questions, adults and children often avert their gaze at certain points within the interaction, especially when questions are difficult (Doherty-Sneddon, Bruce, Bonner, Longbotham, \& Doyle, 2002; Glenberg, Schroeder, \& Robertson, 1998). Gaze aversion may be a way of managing the cognitive load associated with the processing of visual environmental information, or it may serve to alleviate a negative social-emotional experience, such as the self-consciousness associated with, for example, a fear of failure. In the present study, thirty-six 8-year-olds were questioned either face to face or across a live video link. Questions varied in type (arithmetic, verbal reasoning, and autobiographical and episodic memory) and in difficulty. Children averted their gaze more during face-to-face questioning than during video-mediated questioning; however, question difficulty had a very strong influence on aversion in both interview conditions. It is concluded that although social factors play a role in children's gaze aversion during pedagogical question-answer sequences, the primary function of averting gaze is to manage the cognitive load involved in the processing of environmental information.
\end{abstract}

During difficult cognitive activity-for example, remembering information, thinking of an answer to a question, planning what we are going to say, or speaking - we often close our eyes, look up at the sky, or look away from the person we are in conversation with (Doherty-Sneddon, Bruce, Bonner, Longbotham, \& Doyle, 2002; Glenberg, Schroeder, \& Robertson, 1998). A number of studies have reported ways in which adults switch off from environmental stimulation (both live faces and other sorts of visual displays) in order to concentrate on cognitive tasks (e.g., Beattie, 1981; Glenberg et al., 1998). Our own work has shown that older children (8-year-olds) also look away more when answering difficult questions, as compared with easy ones, when questioned face to face (FTF; Doherty-Sneddon et al., 2002; Phelps, DohertySneddon, \& Warnock, in press). Gaze aversion is, therefore, potentially a useful cue during pedagogical interactions, since it gives a nonverbal indication of a child's level of understanding and concentration (see DohertySneddon et al., 2002). This article reports a study of gaze aversion by 8 -year-olds when they were asked questions of increasing difficulty. The children were questioned by the same interviewer either across a live video link (LVL) or FTF, to ascertain what underlies our tendency to avert our gaze when we need to concentrate.

Considerable research effort has been expended on examining the role played by visual communication signals

This work was supported by ESRC Grant R000239930, held by G.D.-S. We thank the children who participated in this research and their teachers and parents for their cooperation and consent. Correspondence regarding this article should be addressed to G. Doherty-Sneddon, Department of Psychology, University of Stirling, Stirling FK9 4LA, Scotland (e-mail: gds1@stir.ac.uk). (e.g., eye gaze, gestures, and facial expressions) in human interaction. There is much evidence that these cues are often important sources of information, and many researchers have proposed that they play a facilitatory role in human communication (e.g., Clark \& Brennan, 1991; Goldin-Meadow, Wein, \& Chang, 1992; McNeill, 1985). However, the fact that such signals are informative means that they carry a cognitive load. The processing costs of visual signals have been documented. Excessive eye gaze between speakers is associated with increased cognitive load, evidenced by, for example, less fluent speech (Beattie, 1981). In addition, the cognitive difficulty of a task relates to the likelihood that people will avert their gaze from other people's faces (Doherty-Sneddon et al., 2002; Ellyson, Dovidio, \& Corson, 1981; Glenberg et al., 1998; Phelps et al., in press). So one explanation of the link between cognitive difficulty and gaze aversion is that the interlocutor's face, an information-rich aspect of the environment, requires cognitive resources to monitor (Glenberg, 1997; Glenberg et al., 1998). When people avert their gaze, they can deploy additional cognitive resources to the task at hand and, hence, improve their performancethe cognitive load hypothesis (cf. Glenberg, 1997).

In addition to the cognitive influences on gaze behavior, social psychologists have drawn attention to the social constraints on gaze in conversation. Gaze serves a number of social functions, including being a signal of intimacy, dominance, and social competence (e.g., Argyle, 1996; Argyle \& Dean, 1965; Burgoon, Manusov, Mineo, $\&$ Hale, 1985). Argyle suggests that gaze indicates intimacy, and levels must be negotiated to maintain a suitable equilibrium in conversation. For example, when speakers are physically close, this leads to a higher level of intimacy that is compensated for by gaze being averted from 
an interlocutor's face. Question topic also influences intimacy distance. Exline, Gray, and Schuette (1965) found that people engaged in less eye contact when asked embarrassing questions than when asked innocuous questions. Therefore, if a question is difficult or personally salient, this may increase self-consciousness or embarrassment, thereby leading to increased gaze aversion to compensate for this. Similarly, Stanley and Martin (1968) proposed that eye contact is associated with an increase in anxiety and that gaze aversion plays a role in anxiety reduction. Part of the load associated with looking at faces, especially when difficult questions are answered, may, therefore, be associated with self-consciousness. It is, therefore, important to understand the relative impact of social and cognitive influences on gaze aversion, since one set of reasons why children avert their gaze during difficult questioning may be because they feel embarrassed or self-conscious and want to avoid looking at the questioner. The question addressed by the present article is not whether gaze aversion per se is influenced by social or cognitive factors, since both clearly contribute to patterns of gaze. The key issue is whether the increase in aversion associated with increasing difficulty of questions is driven primarily by a need to reduce environmental stimulation, especially visual stimulation from the face (faces are a particularly salient type of visual form; Russell \& Lavie, 2001), or to alleviate the selfconsciousness that may accompany being "put on the spot" by difficult questioning.

The study of gaze aversion has important practical applications. For example, Vrij (2002) cited gaze aversion as a nervous behavior commonly believed to be associated with deception. He proposed that people who are intentionally trying to deceive others often feel nervous about being found out and will also have to "think hard" in order to make their lies convincing. In addition, he predicted that younger children are likely to exhibit more signs of hard thinking and nervousness (including more gaze aversion) while lying - in part, because they are less able to control their behavior. However, a number of studies have clearly shown that gaze aversion is not a reliable cue to deception (e.g., Zuckerman \& Driver, 1985). Recent work in which gaze aversion in children has been investigated has shown that young children (5 years and younger) were inconsistent in their use of gaze aversion as a response to cognitive difficulty and often, for example, "stared the adult out" when they experienced difficulties (Doherty-Sneddon et al., 2002). Understanding gaze aversion is, therefore, crucial in order to successfully inform applications of knowledge.

Glenberg et al. (1998) have reported that adults avert their gaze when answering difficult questions even when these are presented on a computer screen, suggesting that gaze aversion functions to reduce environmental stimulation per se, rather than to reduce social factors, such as feeling embarrassed. In the present study, we manipulate social factors by comparing FTF questioning with video-mediated questioning. Evidence that videomediated communication (VMC) has important social effects on users comes from research by Doherty-Sneddon and colleagues. She investigated how children of different ages use verbal and nonverbal signals in FTF and video-mediated interactions (e.g., Doherty-Sneddon \& Kent, 1996; Doherty-Sneddon \& McAuley, 2000). Included in this work was a study of the impact of video links on children when they were questioned as witnesses. In a number of countries, including the United States, Canada, and the United Kingdom, when children are called as witnesses in criminal trials, they are sometimes given the option of being cross-examined via LVLs rather than in open court (Davies, 1999). In a study of real-life court testimony of children, Davies and Noon (1991) found that LVLs reduced stress in child witnesses and improved the quality of their evidence: LVL children were rated as more fluent and audible; they were rated as happier and were more likely to be judged as competent to swear the oath. Doherty-Sneddon and McAuley (2000) found that children experienced an increase in social distance between themselves and the interviewer in VMC that influenced many aspects of their verbal and nonverbal communication (e.g., an increased resistance to misleading questions and a more relaxed demeanor). In other words, the children appeared to be less self-conscious and more socially confident when interviewed across the video link.

These results are consistent with findings from studies of adults using live video links. Overgazing by adult users of VMC has been reported by a number of authors (e.g., Abel, 1990). Indeed, our own research has shown that adults look at one another around 56\% more when communicating via LVLs, as compared with FTF (DohertySneddon et al., 1997). This suggests that the social norms relating to gaze are different when interactions are mediated, even for adults. The present study is novel in that we investigate whether the use of gaze aversion as a cognitive management strategy changes when gaze is video mediated versus FTF.

Of course, as well as increased social distance, there can be an attenuation of visual cues in VMC (see DohertySneddon et al., 1997). In the present study, we used a life-sized head and upper body shot in the mediated condition, so that the size of the mediated face was equivalent to that of the live face, allowing good discrimination of facial features and movement. Furthermore, the mediated condition allowed for mutual eye contact, a very important part of FTF interaction. Although it is possible that physical aspects of the display (e.g., loss of depth cues in a two-dimensional image) meant that the mediated face was a less potent source of visual information, this is unlikely, since a number of researchers have shown that high-quality VMC (e.g., one with no delay in the audio signal) results in the transmission of amounts of information equivalent to those found in FTF interaction (e.g., Anderson et al., 1997). In addition, Bruce (1995) 
reported that perception of facial expressions remains intact in video-mediated interactions.

So gaze aversion, from the face of an interlocutor, occurs more often during cognitively demanding tasks, to reduce cognitive load by reducing visual environmental stimulation and/or to alleviate self-consciousness. In this study, we attempted to tease apart these aspects. We did this by comparing gaze aversion in video-mediated interactions with that in FTF encounters. In the LVL condition, it was assumed (on the basis of previous research) that there would be an increase in social distance between the child and the questioner, reducing the social impact of the questioner's face and changing the norms relating to acceptable levels of gaze. We therefore predicted that the children would avert their gaze less during the video-mediated interactions. Furthermore, if the tendency to avert during difficult questions is due to the self-consciousness associated with being asked difficult questions, we would predict that the difficulty of the questions would have less of an influence on gaze aversion when social presence was alleviated. If, however, children avert their gaze during difficult questions in order to reduce incoming environmental information, question difficulty should influence gaze aversion regardless of whether the interaction was mediated or not.

In this study, we investigated 8-year-olds' gaze aversion. This age group was chosen because it was one that we had previously found to respond to increasing question difficulty by increasing the use of gaze aversion in a way that was equivalent to that for adults. For example, Doherty-Sneddon et al. (2002) found that, like adults, 8 -year-olds looked away more when asked difficult verbal and arithmetic questions. In addition, their gaze aversion peaked while the children were thinking, followed by when they were speaking, with the lowest amount of aversion occurring during listening. This is the same pattern as that found with adults. In contrast, younger children (5-year-olds) apply gaze aversion much less consistently and to a lesser extent. The authors' most recent work in progress suggests that there is an important development of gaze aversion behaviors between 5 and 6 years of age (Phelps et al., in press). By 8 years of age, adult patterns are firmly established. Furthermore, by 8 years, children have acquired considerable knowledge of the social rules about gazing and are likely to use gaze and gaze aversion in response to social situations in ways beginning to be similar to those of adults (Abramovitch \& Daly, 1978). The study of 8-year-olds, therefore, will extrapolate well to older children and adults.

We questioned 8-year-old children either FTF or across an LVL. Questions varied in both type (arithmetic, verbal reasoning, autobiographical memory, or episodic memory) and difficulty (easy, medium, or hard). The hypotheses of this study were the following: (1) We predicted that children would avert their gaze less when interviewed across an LVL, as compared with FTF, and (2) on the basis of our own previous work (DohertySneddon et al., 2002; Phelps et al., in press), we also ex- pected that gaze aversion would be more frequent when the children were asked moderate and very difficult questions, as compared with easy ones. If this functions primarily to alleviate self-consciousness, we would expect to see a greater effect of task difficulty in FTF interaction than in video-mediated questioning. If however, children increase their aversion primarily to switch off from visual environmental stimulation, we would expect an effect of question difficulty in both interview conditions.

\section{METHOD}

\section{Participants}

Thirty-six children (16 boys and 20 girls) took part in the study; their mean age was 8 years 5 months (range $=8$ years 3 months to 9 years 5 months). Children were randomly allocated to one of two interview conditions: 17 were interviewed via an LVL ( 7 boys and 10 girls; $M$ age $=8$ years 5 months, range $=8$ years 4 months to 9 years 4 months) and 19 were interviewed FTF ( 9 boys and 10 girls; $M$ age $=8$ years 4 months, range $=8$ years 3 month to 9 years 5 months). A further 2 children were allocated to the LVL condition. Of these, 1 chose not to participate; the data for the other child were lost, owing to equipment failure. Unpaired comparisons showed that the groups were comparable in terms of their age $[t(34)=0.56$, $p=.28]$. Inclusion criteria specified English as the first language. None had previously participated in related experiments.

\section{Stimuli}

Four different types of questions were posed to the children: verbal $(n=36)$, mental arithmetic $(n=36)$, episodic memory $(n=$ $18)$, and autobiographical memory $(n=18)$. Both the verbal questions and the arithmetic questions were based on stimuli described in the Wechsler Preschool and Primary Scale of Intelligence, where verbal questions required the children to define words $(n=9)$, spell words $(n=9)$, repeat word lists $(n=9)$, and give information about concepts $(n=9)$. The mental arithmetic questions involved addition $(n=9)$, subtraction $(n=9)$, multiplication/division $(n=9)$, and number use $(n=9)$. The autobiographical questions involved asking the children to recollect events that they had personally experienced, such as information pertaining to recent classroom activities. The episodic memory questions were based on stimuli described in the Wechsler Memory Scale-Revised (WMS-R). This involved instructing the children to remember stimuli paired in time (e.g., car-red or ball-green). The experimenter then provided one half of each pairing (e.g., "what color was the car?"), and the child's task was to correctly recollect the event with which it had previously been paired (i.e., red).

For each type of question, there were three levels of difficulty: easy, medium, and hard. These levels of difficulty were deemed to be appropriate through consultation with the participants' teachers $(n=3)$, who rated how difficult an average 8-year-old would find each question. Rating instructions stressed that the questions would be read aloud to the child, who, in turn, would give a verbal response. Teachers rated the difficulty of an initial set of 232 questions, using a 7-point scale, with 1 labeled very easy, through 4 labeled moderately easy, through 7 labeled very hard. These ratings were used to establish the mean difficulty value for each question, and enabled the compilation of a controlled set of questions $(n=$ 108 ), with questions falling under three distinct levels of difficulty: easy (mean difficulty score ranging from 1.0 to 2.0), medium (mean difficulty score ranging from 3.5 to 4.5 ), and hard (mean difficulty score ranging from 6.0 to 7.0 ). Teachers' ratings for the difficulty levels of critical stimuli correlated highly [Teacher 1 vs. Teacher 2, $r(107)=.86$; Teacher 1 vs. Teacher $3, r(107)=.92$; Teacher 2 vs. Teacher 3, $r(107)=.91]$. Examples of easy, medium, and hard verbal-vocabulary questions were What is a photograph? What is 
a menu? and What is humor? respectively. Examples of the different levels of arithmetic questions were: $9-6=\ldots, 30-12=\ldots$, and $265-34=\ldots$. Easy, medium, and hard autobiographical questions were, for example, "How many points did your class group get: this fortnight; last fortnight; two fortnights ago?" Easy episodic questions involved the child's remembering two different pairings of information, medium questions involved three pairings, and hard questions involved four.

\section{Apparatus}

Questions were asked either FTF or via LVL. In the case of the LVL, images of the face and upper body were transmitted through the use of video tunnels (Smith, O'Shea, O'Malley, Scanlon, \& Taylor, 1991). Each of these consisted of a color monitor (JVC TM90PSN, $35 \mathrm{~cm}$ diagonally) mounted in a wooden box behind a halfsilvered mirror. A second, fully silvered mirror was fixed parallel above the half-silvered mirror, so that light reflected from the first mirror would reflect off the second into a video camcorder (Panasonic M10) located behind the box and above the TV monitor. Because of the half-silvered nature of the first mirror, the child, looking directly into the video tunnel, was able to see an image of the experimenter on the monitor. The child's image was also reflected off the mirror into a second camcorder, from which it was transmitted to the monitor in the experimenter's identically arranged video tunnel. With correct positioning of the camera, the child and the experimenter were able to make direct eye contact with each other. Both the child and the experimenter were seated directly in front of the video tunnels.

\section{Procedure}

The children were asked the questions individually in a quiet location separate from their classroom. The ordering of question type, question difficulty, and the individual questions was fully counterbalanced across participants. Questioning took approximately $35 \mathrm{~min}$ for each child. The children interviewed FTF were seated directly facing the questioner across a table (at a distance of approximately $5 \mathrm{ft}$ ) and were, therefore, able to see the face and upper body of the questioner. The children interviewed via an LVL were also seated directly facing the questioner (again at a distance of approximately $5 \mathrm{ft}$ ), but in this instance, they saw one another on a TV monitor. The image also showed a head and upper body shot, with the face life-sized. The amount of the questioner the child could see was, therefore, roughly equivalent across conditions. In both conditions, the questioner maintained her gaze on the child's face throughout each question episode-from when she began speaking the question until the child finished responding.

To enable quantification of the children's direction of eye gaze during each stage of the question-answer interaction (listening, thinking, and speaking), a front-on view of each child's head and shoulders was video recorded throughout testing, using a digital camcorder. Although all the children were fully aware that they were being filmed, they were not aware that their gaze behavior was of specific interest.

The dependent measures were (1) response accuracy for each type of question and (2) the mean percentage of time that gaze was averted during the thinking stage of the interaction for each type of question. The amount of time gaze was averted was measured from when the experimenter stopped speaking the question until the child started the response and included pauses, hesitations, and requests for the question to be repeated. This time was converted to percentage of time spent averting gaze by dividing the gaze avert time by the total thinking time and multiplying by 100 . The children did avert their gaze while speaking their responses, but this was the case for less than half the amount of time, as compared with thinking (mean thinking gaze aversion $=61.2 \%$, mean speaking gaze aversion $=29.0 \%$ ). Thinking time was of particular interest in this study, since our earlier work had shown this to be the period of highest cognitive load and that in which gaze aversion is most frequent
(Doherty-Sneddon et al., 2002), and was the period chosen for analysis.

Interjudge reliability as to whether gaze aversion had occurred was calculated for a random sample of $11.11 \%$ of the participants. This calculation included all of the listening, thinking, and speaking aversion scores for every question asked. In total, 1,296 episodes were coded by two judges, for which there was $87.04 \%$ interjudge agreement. Furthermore, the coders' scoring for the duration of gaze aversion correlated significantly $[r(1,295)=.62, p<.01]$.

\section{RESULTS}

The effects of question difficulty and interview status on both response accuracy and the percentage of time that gaze was averted during the thinking stage of the interaction (the period of time from when the questioner stopped speaking until the child began to speak) were analyzed as described in the following sections.

\section{Accuracy of Response}

We made no predictions about accuracy of responses to questions across conditions. Analyses of accuracy were done to show that the three levels of difficulty were set appropriately. For each type of question, a 4 (question type: autobiographical memory, arithmetic, verbal, or episodic memory) $\times 3$ (question difficulty: easy, medium, or hard) $\times 2$ (interview status: FTF or LVL) mixed design analysis of variance (ANOVA) was employed, with question type and question difficulty the within-groups variables and interview status the between-groups variable. Means for each condition are displayed in Table 1.

There was a significant effect of question type $[F(3,99)=7.41, p<.0001]$. Paired $t$ tests showed that there was greater accuracy for the episodic memory questions and the arithmetic questions than for both the verbal questions $[t(34)=3.63, p<.001$, and $t(35)=4.07, p<$ .001 , respectively] and the autobiographical questions $[t(34)=3.38, p<.01$, and $t(35)=2.69, p<.01$, respectively; means: episodic $=69.84 \%$, arithmetic $=67.70 \%$, autobiographical $=60.77 \%$, and verbal $=60.15 \%]$. There was a significant effect of question difficulty $[F(2,66)=$ 401.33, $p<.0001]$. Paired $t$ tests showed that there was greater accuracy for easy questions than for both medium questions $[t(35)=12.42, p<.0001]$ and hard questions $[t(35)=29.02, p<.0001]$ and for medium questions than for hard questions $[t(35)=16.84, p<.0001$; means: easy $=88.15 \%$, medium $=64.54 \%$, and hard $=41.20 \%]$. There was no effect of interview status. There was a significant interaction between question type and question difficulty $[F(6,198)=6.53, p<.0001]$. Simple effects analyses showed that when questions were easy, there was greater accuracy for arithmetic questions than for all other types of questions (autobiographical memory questions, $p<.0001$; verbal questions, $p<.05$; episodic memory questions, $p<.01]$. When questions were medium, there was greater accuracy for arithmetic questions than for autobiographical memory questions $(p<.05)$ and verbal questions ( $p<.01$ only). When questions were hard, there was greater accuracy for episodic memory questions than for all other types of questions (autobio- 
Table 1

Mean Percentages for Accuracy of Responses to Different Question Types for the Live Video Link (LVL) Group and the Face-to-Face (FTF) Group (With Standard Deviations)

\begin{tabular}{|c|c|c|c|c|c|c|c|c|c|c|c|c|}
\hline \multirow[b]{4}{*}{ Question Type } & \multicolumn{12}{|c|}{ Question Difficulty } \\
\hline & \multicolumn{6}{|c|}{ LVL Group } & \multicolumn{6}{|c|}{ FTF Group } \\
\hline & \multicolumn{2}{|c|}{ Easy } & \multicolumn{2}{|c|}{ Medium } & \multicolumn{2}{|c|}{ Hard } & \multicolumn{2}{|c|}{ Easy } & \multicolumn{2}{|c|}{ Medium } & \multicolumn{2}{|c|}{ Hard } \\
\hline & $M$ & $S D$ & $M$ & $S D$ & $M$ & $S D$ & $M$ & $\overline{S D}$ & $M$ & $S D$ & $M$ & $S D$ \\
\hline Autobiographical memory & 78.96 & 15.48 & 61.25 & 16.64 & 38.96 & 11.66 & 87.89 & 11.86 & 59.56 & 17.94 & 36.05 & 16.20 \\
\hline Arithmetic & 97.40 & 5.02 & 70.17 & 17.70 & 40.10 & 13.68 & 91.15 & 7.69 & 70.14 & 17.37 & 36.36 & 22.49 \\
\hline Verbal & 89.32 & 20.69 & 65.68 & 30.97 & 39.84 & 22.67 & 87.94 & 9.71 & 53.29 & 18.30 & 26.10 & 14.82 \\
\hline Episodic memory & 82.29 & 22.13 & 68.40 & 16.57 & 55.21 & 16.56 & 88.16 & 13.12 & 68.71 & 15.85 & 55.70 & 14.38 \\
\hline
\end{tabular}

graphical memory questions, $p<.0001$; arithmetic questions, $p<.0001$; verbal questions, $p<.0001)$. No further interactions were found.

\section{Gaze Aversion During Thinking Stage}

A 4 (question type: autobiographical memory, arithmetic, verbal, or episodic memory) $\times 3$ (question difficulty: easy, medium, or hard) $\times 2$ (interview status: FTF or LVL) mixed design ANOVA was employed, with question type, question difficulty, and episode the withingroups variables and interview status the between-groups variable. Means for each condition are displayed in Table 2.

There was a significant effect of question type $[F(3,99)=30.09, p<.0001]$. Paired $t$ tests showed that the children used significantly more aversion for autobiographical memory questions than for all other types of questions [arithmetic $[t(35)=4.48, p<.0001$; verbal $[t(35)=9.64, p<.0001$; episodic memory $[t(34)=6.84$, $p<.0001]$. The children also used significantly more aversion for arithmetic questions than for verbal questions $[t(35)=5.40, p<.0001]$ and episodic memory questions $[t(34)=2.67, p<.01]$ and for episodic memory questions than for verbal questions $[t(34)=2.00$, $p=.054$; means: autobiographical memory $=71.76 \%$, arithmetic $=62.77 \%$, episodic memory $=57.86 \%$, and verbal $=53.60 \%]$. There was a significant effect of question difficulty $[F(2,66)=156.04, p<.0001]$. Paired $t$ tests showed that children used significantly more aversion for both hard questions $[t(35)=14.31, p<.0001]$ and medium questions $[t(35)=14.38, p<.0001]$ than for easy questions and for hard questions than for medium questions $[t(35)=4.35, p<.0001$; means: easy $=46.10 \%$, medium $=67.03 \%$, hard $=72.26 \%$. There was a signif- icant effect of interview status $[F(1,33)=4.58, p<.05]$, with the children using significantly more aversion when interviewed FTF (means: FTF $=65.86 \%, \mathrm{LVL}=56.59 \%$ ). There was a significant interaction between question type and question difficulty $[F(6,198)=11.17, p<$ $.0001]$. Simple effects analyses showed that although there was significantly more aversion for both hard questions and medium questions than for easy questions for each type of question (i.e., autobiographical memory, arithmetic, verbal, and episodic memory), it was only for the verbal questions and episodic memory questions that there was also significantly more aversion for hard questions than for medium questions (all $p$ s $<.0001$ ). No further interactions were found. Importantly, the interaction between question difficulty and interview status was not significant $[F(2,66)=0.082, p=.92$; mean $\mathrm{FTF}$, easy $=$ 50.3 , medium $=70.6$, hard $=76.7$; mean LVL, easy $=$ 40.7 , medium $=62.1$, hard $=67.0]$. In the FTF condition, the $95 \%$ confidence interval (CI) for the easy-hard difference was $26.37 \pm 5.13$, and in the LVL condition, it was $25.94 \pm 5.09$. These differences are large relative to their CIs, and it follows that the only interactions that could plausibly be present are substantially smaller than the differences between the easy and the hard tasks.

\section{DISCUSSION}

For all types of questions, the children averted their gaze less when interviewed across an LVL, as compared with FTF, even though the actual physical distance between the experimenter and the child was held constant across interview conditions and the amount of the questioner visible was equivalent. Furthermore, the children

Table 2

Mean Percentages of Time Spent in Gaze Aversion While Thinking by the Live Video Link (LVL) Group and the Face-to-Face (FTF) Group (With Standard Deviations)

\begin{tabular}{|c|c|c|c|c|c|c|c|c|c|c|c|c|}
\hline \multirow[b]{4}{*}{ Question Type } & \multicolumn{12}{|c|}{ Question Difficulty } \\
\hline & \multicolumn{6}{|c|}{ LVL Group } & \multicolumn{6}{|c|}{ FTF Group } \\
\hline & \multicolumn{2}{|c|}{ Easy } & \multicolumn{2}{|c|}{ Medium } & \multicolumn{2}{|c|}{ Hard } & \multicolumn{2}{|c|}{ Easy } & \multicolumn{2}{|c|}{ Medium } & \multicolumn{2}{|c|}{ Hard } \\
\hline & $M$ & $S D$ & $M$ & $S D$ & $M$ & $S D$ & $M$ & $S D$ & $M$ & $S D$ & $M$ & $S D$ \\
\hline Autobiographical memory & 53.69 & 17.09 & 71.11 & 14.87 & 72.06 & 16.36 & 68.25 & 15.55 & 81.21 & 11.18 & 80.45 & 11.70 \\
\hline Arithmetic & 35.04 & 17.09 & 68.47 & 19.39 & 69.56 & 23.73 & 48.09 & 24.80 & 72.97 & 17.24 & 81.67 & 10.01 \\
\hline Verbal & 40.98 & 14.97 & 50.87 & 15.59 & 57.86 & 13.25 & 43.42 & 18.08 & 60.79 & 18.89 & 68.03 & 15.64 \\
\hline Episodic memory & 33.17 & 23.02 & 57.83 & 17.88 & 68.47 & 12.05 & 41.46 & 21.22 & 67.46 & 14.41 & 76.55 & 11.78 \\
\hline
\end{tabular}


averted their gaze most when being asked personally relevant questions - the autobiographical memory questions. This could in part be because the autobiographical questions were more difficult (shown by the decreased accuracy of response, as compared with episodic and arithmetic questions). However, verbal and autobiographical questions had equivalent accuracy levels, and still the autobiographical questions elicited more gaze aversion than did the verbal. Clearly, there is a significant social component driving interpersonal gaze aversion during questioning. Part of the reason we look away in order to concentrate could be because we experience a negative social emotion, such as self-consciousness or embarrassment (Stanley \& Martin, 1968). An alternative social explanation is that we use gaze aversion to deliberately signal to the other person that we are in the process of thinking. Some recent doctoral work suggests that children can deliberately use gaze aversion as a signal of thinking. For example, children and adults are more likely to look $u p$ while thinking if their questioner is facing them but to look down if the questioner is turned away from them (McCarthy, 2004). In a similar vein, Kendon (1967) reported that adults use looking away as a deliberate turn-holding cue-in other words, as a way of saying "I'm about to say something"/“I'm still thinking about what I'm going to say."

The present results make sense in terms of our own earlier work. We have shown that the reduction in social co-presence produced by video-mediated interviews results in children being more confident and less intimidated. This had an important impact on many aspects of their verbal and nonverbal behavior; for example, they were more relaxed and happy and better able to disagree with an adult questioner when asked misleading questions (Doherty-Sneddon \& McAuley, 2000). The data are also consistent with our research with adults who look at one another around 56\% more when communicating via LVLs, as compared with FTF (Doherty-Sneddon et al., 1997). So children and adults reduce the baseline amount that they avert their gaze from the face of an interlocutor when they communicate across LVLs. Our next question was whether cognitive difficulty of questions would influence gaze aversion in both mediated and FTF conditions equally.

Earlier work (Doherty-Sneddon et al., 2002; Phelps et al., in press) has shown that gaze aversion is more frequent when children are asked moderately difficult questions than when asked easy ones. If this is due primarily to the self-consciousness associated with dealing with cognitively difficult material, we would expect to see a greater effect of task difficulty in FTF interaction and little effect in video-mediated questioning. If, however, children increase their aversion to avoid environmental information (in this case, the face of the questioner), we would expect an effect of question difficulty in both interview conditions. Our results support the latter possibility. Question difficulty had an enormous impact on the amount that the children averted their gaze from the experimenter's face in both interview contexts. Furthermore, this was consistently the case across all question types. The children averted their gaze, on average, 52\% more when answering hard questions, as compared with their easy counterparts, in FTF interviews. Similarly, they increased their gaze aversion by $64 \%$ for hard questions in the LVL condition. Even when social factors were greatly reduced (LVLs), the children looked away from their interviewer more when they were trying to process increasingly difficult information. This gives considerable weight to the cognitive load hypothesis. The processing costs of visual communication signals (such as facial expressions, eye gaze, and lip movements) are documented. For example, if one is forced to look constantly at a listener while speaking, one's speech will become less fluent and contain more "erms" and "uhms" (Beattie, 1981). We have found that asking children to maintain gaze with a speaker's face while listening to descriptions of abstract shapes interferes with their abilities to understand these descriptions. In addition, we found that children are less able to retain visuospatial information when they have to monitor a face during retention than if they are allowed to look away (DohertySneddon, Bonner, \& Bruce, 2001). So gaze aversion is an overt strategy for shifting one's attention from environmental stimulation (such as faces) to processing of other internal information. The present study shows that this is true for both live and video-mediated faces.

This work has clear educational implications. It appears that children use gaze aversion to control their own cognitive load. There are social factors at work, and teachers and others working with children would be well advised to allow children to set a comfortable intimacy distance and avoid intimidating questioning. Another practical application of this work is with child witnesses. Earlier work has shown that children are more confident and less suggestible to misleading questions when crossexamined across LVLs (e.g., Davies, 1999). The fact that the children in the present study averted their gaze from the questioner less during video link interactions suggests that they too were less intimidated. It is yet to be established whether the gazing behavior of child witnesses, either in open court or across LVLs, influences how credible jurors perceive them to be, an extremely important issue for the court process (Goodman et al., 1998).

Cultural rules about gaze behavior also help determine our response to gaze and gaze aversion. For example, in Britain, children are typically told to "Look at me when I'm speaking to you," whereas in America, children are encouraged "Not to stare." In addition, Black Americans interpret gaze aversion as a sign of respect, rather than as a sign of disinterest (Hanna, 1984). Cultures differ significantly in terms of how much gaze between individuals is acceptable or desirable. For example, with contact cultures (such as Latin America and Southern Europe), people engage in more gaze, overall, than do people coming from noncontact cultures, such as Northern Europe (Halberstadt, 1985; Watson, 1970). 
However, social influences aside, we must also be aware of the important cognitive function that gaze aversion plays in any conversation, especially when the material being discussed is demanding. Gaze aversion is often a sign of concentration and inner effort. Teachers and other professionals must be proficient in reading children's gaze aversion in conjunction with other cues, in order to determine, for example, when children are engaged in learning, are just about to understand, or perhaps are switching off from a task (Doherty-Sneddon et al., 2002). Indeed, in our ongoing research, we are investigating whether teachers do, in fact, use information from children's gaze (Doherty-Sneddon \& Phelps, 2005).

\section{REFERENCES}

Abel, M. (1990). Experiences in an exploratory distributed organization. In J. Gallagher, R. E. Kraut, \& C. Egido (Eds.), Intellectual teamwork: Social and technological foundations of cooperative work (pp. 489-510). Hillsdale, NJ: Erlbaum.

Abramovitch, R., \& Daly, E. M. (1978). Children's use of head orientation and eye contact in making attributions of affiliation. Child Development, 49, 519-522.

Anderson, A. H., O'Malley, C., Doherty-Sneddon, G., Langton, S., Newlands, A., Mullin, J., Fleming, A. M., \& Van der Velden, J. (1997). The impact of VMC on collaborative problem solving: An analysis of task performance, communicative process, and user satisfaction. In K. E. Finn, A. J. Sellen, \& S. B. Wilbur (Eds.), Videomediated communication (pp. 133-156). Mahwah, NJ: Erlbaum.

Argyle, M. (1996). Bodily communication. London: Routledge.

Argyle, M., \& Dean, J. (1965). Eye-contact, distance and affiliation. Sociometry, 6, 289-304.

Beattie, G. W. (1981). A further investigation of the cognitive interference hypothesis of gaze patterns during conversation. British Journal of Social Psychology, 20, 243-248.

BRUCE, V. (1995). The role of the face in face-to-face video communication. In S. J. Emmott (Ed.), Information superhighways: Multimedia users and futures (pp. 227-238). London: Academic Press.

Burgoon, J. K., Manusov, V., Mineo, P., \& Hale, J. L. (1985). Effects of gaze on hiring, credibility, attraction and relational message interpretation. Journal of Nonverbal Behavior, 9, 133-146.

ClaRK, H. H., \& BRENNAN, S. E. (1991). Grounding in communication. In L. B. Resnick, J. Levine, \& S. D. Teasley (Eds.), Perspectives on socially shared cognition (pp. 127-149). Washington, DC: American Psychological Association.

DAVIES, G. (1999). The impact of television on the presentation and reception of children's testimony. International Journal of Law \& Psychiatry, 22, 241-256.

DAVIES, G., \& NoON, E. (1991). An evaluation of the live link for child witnesses [Home Office report]. London.

Doherty-Sneddon, G., Anderson, A. H., O'Malley, C., Langton, S., Garrod, S., \& Bruce, V. (1997). Face-to-face and video mediated communication: A comparison of dialogue structure and task performance. Journal of Experimental Psychology: Applied, 3, 105-125.

Doherty-SNedDON, G., Bonner, L., \& Bruce, V. (2001). Cognitive demands of face monitoring: Evidence for visuospatial overload. Memory \& Cognition, 29, 909-919.

Doherty-Sneddon, G., Bruce, V., Bonner, L., Longbotham, S., \& Doyle, C. (2002). Development of gaze aversion as disengagement from visual information. Developmental Psychology, 38, 438-445.

Doherty-Sneddon, G., \& Kent, G. (1996). Visual signals and the communication abilities of children. Journal of Child Psychology \& Psychiatry, 37, 949-959.
Doherty-Sneddon, G., \& McAuley, S. (2000). Influence of video mediation on adult-child interviews: Implications for the use of the live link with child witnesses. Applied Cognitive Psychology, 14, 379-392.

DoherTy-SNeddon, G., \& PhelPs, F. (2005). Teachers' responses to children's gaze aversion during classroom interactions. Manuscript in preparation.

Ellyson, S. L., Dovidio, J. F., \& Corson, R. L. (1981). Visual behavior differences in females as a function of self-perceived expertise. Journal of Nonverbal Behavior, 5, 164-171.

Exline, R., Gray, D., \& Schuette, D. (1965). Visual behavior in a dyad as affected by interview content and sex of respondent. Journal of Personality \& Social Psychology, 1, 201-209.

Glenberg, A. M. (1997). What memory is for. Behavioral \& Brain Sciences, 20, 1-19.

Glenberg, A. M., Schroeder, J. L., \& Robertson, D. A. (1998). Averting the gaze disengages the environment and facilitates remembering. Memory \& Cognition, 26, 651-658.

Goldin-Meadow, S., Wein, D., \& Chang, C. (1992). Assessing knowledge through gesture: Using children's hands to read their minds. Cognition \& Instruction, 9, 201-219.

Goodman, G. S., Tobey, A. E., Batterman-Faunce, J. M., Orcutt, H., Thomas, S., Shapiro, C., \& Sachsenmaier, T. (1998). Face-to-face confrontation: Effects of closed-circuit technology on children's eyewitness testimony and jurors' decisions. Law \& Human Behavior, 22, 165-203.

HALBERSTADT, A. G. (1985). Differences between blacks and whites in nonverbal communication. In A. W. Siegman \& S. Feldstein (Eds.), Multichannel integrations of nonverbal behavior (pp. 229-259). Hillsdale, NJ: Erlbaum.

HANNA, J. L. (1984). Black/white non-verbal differences, dance and dissonance. In A. Wolfgang (Ed.), Nonverbal behavior: Perspectives, applications, intercultural insights (pp. 373-409). Lewiston, NY: Hogrefe.

Kendon, A. (1967). Some functions of gaze direction in social interaction. Acta Psychologica, 26, 22-63.

McCarthy, A. (2004, January). Are the eyes the windows to the soul? Talk given at University of Kyoto, Japan.

McNeILL, D. (1985). So you think gestures are nonverbal? Psychological Review, 92, 350-371.

PhelPs, F., DOHERTY-SNEDDON, G., \& WARNOCK, H. (in press). Functional benefits of children's gaze aversion during questioning. British Journal of Developmental Psychology.

Russell C., \& Lavie, N. (2001). Changing faces: A detection advantage in the flicker paradigm. Psychological Science, 12, 94-99.

Smith, R., O'Shea, T., O'Malley, C., Scanlon, E., \& Taylor, J. (1991). Preliminary experiments with a distributed, multi-media, problem-solving environment. In J. Bowers \& S. Benford (Eds.), Studies in computer-supported co-operative work: Theory, practice and design (pp. 31-48). Amsterdam: Elsevier.

Stanley, G., \& Martin, D. S. (1968). Eye-contact and the recall of material involving competitive and noncompetitive associations. Psychonomic Science, 13, 337-338.

VRIJ, A. (2002). Deception in children: A literature review and implications for children's testimony. In H. L. Westcott, G. M. Davies, \& R. H. C. Bull (Eds.), Children's testimony (pp. 175-194). London: Wiley.

Watson, O. M. (1970). Proxemic behaviour: A cross-cultural study. The Hague: Mouton.

ZuCKERMAN, M., \& DrIVER, R. E. (1985). Telling lies: Verbal and nonverbal correlates of deception. In A. W. Siegman \& S. Feldstein (Eds.), Multichannel integrations of nonverbal behavior (pp. 129147). Hillsdale, NJ: Erlbaum.

(Manuscript received March 5, 2004; revision accepted for publication July 22, 2004.) 